\title{
Arterial Wall Stress Induces Phenotypic Switching of Arterial Smooth Muscle Cells in Vascular Remodeling by Activating the YAP/TAZ Signaling Pathway
}

\author{
Yongshun Wang a,b,c Wei Cao ${ }^{a, b}$ Jinjin Cui ${ }^{a, b}$ Yang Yu ${ }^{a, b} \quad$ Yubo Zhao $^{d}$ Jian Shie \\ Jian Wu ${ }^{a, b}$ Zhengyuan Xia ${ }^{f}$ Bo Yu ${ }^{a, b}$ Jingjin Liu ${ }^{a, b, f}$ \\ aCardiology Department, Second Affiliated Hospital of Harbin Medical University, Harbin, Heilongjiang, \\ ${ }^{b} K e y$ Laboratories of the Education Ministry for Myocardial Ischemia Mechanisms and Treatment, \\ Harbin, Heilongjiang, 'Department of Biomedical Science, University of Hong Kong, Pokfulam, Hong \\ Kong, 'Department of Vascular Surgery, Second Affiliated Hospital of Harbin Medical University, \\ Harbin, Heilongjiang, e'Department of Cardiology, Eighth Affiliated Hospital, Sun Yat-Sen University, \\ Guangzhou, Guangdong, 'Department of Anesthesiology, University of Hong Kong, Pokfulam, Hong \\ Kong, China
}

\section{Key Words}

Vascular Remodeling • Arterial Smooth Muscle Cells • YAP/TAZ • Shear Stress • Cyclic Strain

\begin{abstract}
Background/Aims: Increasing wall stress or biomechanical stretch experienced by arteries influences the initiation of atherosclerotic lesions. This initiation is mediated by Yes-associated protein (YAP) and transcriptional co-activator with PDZ-binding motif (TAZ), which are both effectors of the Hippo pathway. In this study, the functional roles of YAP/TAZ proteins in the regulation of the stretch-mediated programing of human umbilical arterial smooth muscle cells (HUASMCs) to a proliferative phenotype were examined. Methods: HUASMCs were seeded on a Matrigel-coated silicone chamber and subjected to biomechanical stretch for 24 $\mathrm{h}$ after $48 \mathrm{~h}$ of growth. YAP/TAZ small interfering RNA was used to specifically knockdown YAP/ TAZ expression in HUASMCs. Results: We observed that YAP/TAZ activation via biomechanical stretching is involved in the regulation of critical aspects of the HUASMC phenotypic switch. YAP/TAZ knockdown significantly attenuated the stretch-induced proliferative and proinflammatory phenotypes in HUASMCs. Furthermore, treatment with atorvastatin, an antiatherosclerotic drug, attenuated the stretch-induced phenotypic switch of HUASMCs from the contractile to synthetic state by suppressing YAP/TAZ expression. Additional investigations demonstrated the role of stretch in inhibiting the Hippo pathway, leading to the activation of PI3-kinase (PI3K) and phosphoinositide dependent kinase (PDK1); the key molecule for the regulation of the PDK1 and Hippo complex interaction was Sav1. These results showed
\end{abstract}




\section{Cellular Physiology Cell Physiol Biochem 2018;51:842-853

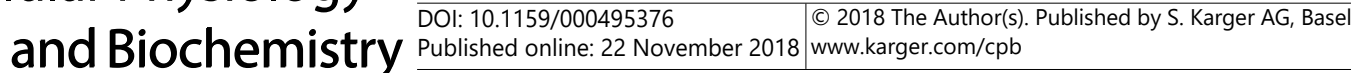 \\ Wang et al.: Arterial Wall Stress Induces Phenotypic Switching of Arterial Smooth \\ Muscle Cells}

the importance of YAP/TAZ activation, induced by biomechanical stretch, in promoting atheroprone phenotypes in HUASMCs. Conclusion: Taken together, our findings revealed a mechanism by which YAP/TAZ activation contributes to the pathogenesis of atherosclerosis.

(C) 2018 The Author(s)

Published by S. Karger AG, Basel

\section{Introduction}

Vascular smooth muscle cells (VSMCs) are essential for maintaining vessel homeostasis. These cells are capable of proliferating and expressing different phenotypes when exposed to alterations in environmental and hormonal stimuli, such as changes in cytokine stimulation, cell adhesion, injuries to vessels, and increased mechanical force [1]. Changes in biomechanical force are especially important with respect to vascular biology; they affect the arterial and venal cells under both physiological and pathophysiological conditions. Despite multiple studies demonstrating associations between specific biomechanical force alterations with vascular remodeling, the mechanisms underlying these associations are still unclear [2-4].

Shear stress and cyclic strain are the two primary mechanical forces normally experienced by large arteries [5]. The endothelium experiences shear stress as blood flows through the lumen as well as cyclic strain; the latter is also experienced by VSMCs. It should be noted that cyclic strain is a major determinant of both normal VSMC physiology and pathophysiology, playing a crucial role in hypertension, vein-graft intimal hyperplasia and failure, restenosis, and atherosclerosis [6]. Indeed, growing evidence indicates that cyclic strain modulates VSMC functions in the media of the arterial wall [7]. The chronic elevation of strain levels, such as in hypertension, stimulates VSMCs to mediate vascular remodeling [8, 9]. This process requires VSMC proliferation, remodeling, and migration, which necessitates the ability to sense and adapt to mechanical stresses. Therefore, a key topic regarding the cyclic strain response is the molecular bases for the capacities of VMSCs to sense and adapt to stress as well as the resulting alterations in gene regulation and signaling via downstream effectors.

One of the signaling pathways involved in mediating the VMSC stretch response is the Hippo signaling pathway, an evolutionarily conserved pathway that inhibits cell proliferation. Indeed, other researchers have shown that a loss of Hippo signaling leads to organ overgrowth and cancer development [10-13]. However, the activation of this pathway leads to the phosphorylation and subsequent cytoplasmic retention of the downstream effectors Yes-associated protein (YAP) and transcriptional co-activator with PDZ-binding motif (TAZ), thereby inhibiting their activity [14]. These effectors are mechanical stimuli sensors, especially with respect to matrix stiffness, stretch, and cell density [15].

However, the exact role of YAP/TAZ in cell stretch-mediated vascular remodeling is unclear. The well-characterized YAP/TAZ target genes CTGF and CYR61 are highly expressed in human atherosclerotic lesions but not in normal human arteries [16]. Additionally, lysophosphatidic acid, a major atherogenic factor, is a potent YAP/TAZ activator [17]. Other biochemical factors, such as cytokines, growth factors, and drugs, are able to regulate YAP/ TAZ activity and the resulting functional outcomes [18-21]. Moreover, YAP has been suggested to play a role in mediating vascular remodeling in carotid stenosis progression [22]. In fact, the strongest YAP inhibitors are the well-known anti-atherosclerotic drugs statins [20].

Given the demonstration that YAP is a potent cell proliferation and migration factor, we hypothesized that YAP plays an important role in the phenotypic switching of VSMCs, which may result in vascular remodeling. The purposes of this research were to test the hypothesis that arterial wall stress and stretch, produced by intraluminal pressure, contribute to the VSMC phenotypic switch and to investigate the underlying mechanisms. 


\section{Cellular Physiology Cell Physiol Biochem 2018;51:842-853 \begin{tabular}{l|l|l} 
and Biochemistry Published online: 22 November 2018 & $\begin{array}{l}\text { (c) } 2018 \text { The Author(s). Published by S. Karger AG, Basel } \\
\text { www.karger.com/cpb }\end{array}$ \\
\hline
\end{tabular} \\ Wang et al.: Arterial Wall Stress Induces Phenotypic Switching of Arterial Smooth \\ Muscle Cells}

\section{Materials and Methods}

\section{Cell culture and treatment}

Human umbilical arterial smooth muscle cells (HUASMCs) were purchased from the American Type Culture Collection (Manassas, VA), and were grown in accordance with the manufacturer's specifications. Cells were cultured in Dulbecco's modified Eagle's medium containing 10\% fetal bovine serum (FBS), 50 $\mathrm{U} / \mathrm{mL}$ penicillin, and $50 \mu \mathrm{g} / \mathrm{mL}$ streptomycin and fungizone (Invitrogen, Carlsbad, CA). These cells were then seeded on a Matrigel-coated silicone chamber at a density of $2 \times 10^{4} \mathrm{cells} / \mathrm{cm}^{2}$. Biomechanical stretch was applied using a Flexcell FX-5000 tension system at a frequency of $0.5 \mathrm{~Hz}$, with elongation from $0 \%$ to $13 \%$, for $24 \mathrm{~h}$ after $48 \mathrm{~h}$ of growth. Cells were also exposed to the following reagents: $20 \mu \mathrm{M}$ SP 600125

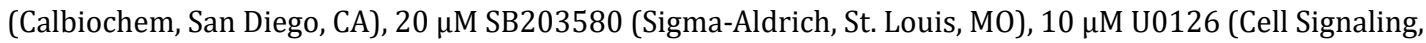
Danvers, MA), $4 \mu \mathrm{M}$ LY294002 (Calbiochem), $20 \mu \mathrm{M}$ AKT inhibitor II (Calbiochem), and $5 \mu \mathrm{M}$ PDK1 inhibitor (Sigma-Aldrich).

\section{Transfection with small interfering RNA}

For gene silencing by small interfering RNA (siRNA), a small double-stranded RNA is used to target mRNAs for degradation. The YAP/TAZ-siRNA duplexes were synthesized by Shanghai GenePharma Co., Ltd. (Shanghai, China) as follows: siRNA-YAP: 5'-UUCUUUAUCUAGCUUGGUGGC-3'; siRNA-TAZ: 5'-CAGCCAAAUCUCGUGAUGAAU- ${ }^{\prime}$; $\quad$ siRNA-Mst1: $\quad 5^{\prime}$-GCUUGCAUGAGGACUACAGAU- $3^{\prime}$; $\quad$ siRNA-SAV1: $5^{\prime}$-GCUUGCAUGAGGACUACAGAU- $3^{\prime}$. Control group cells were treated with either transfection reagents (vehicle) or with non-targeting siRNA (siRNA-NC: 5'-UUCUCCGAACGUGUCACG-3'). Cells were transfected with $200 \mathrm{nM}$ siRNA using the X-treme siRNA Transfection Reagent (Roche Applied Science, Penzberg, Germany) in accordance with the manufacturer's instructions.

\section{Cell proliferation assay}

HUASMC proliferation was determined by 5-ethynyl-2-deoxyuridine (EdU) incorporation (RiboBio, Guangzhou, China). Cells were fixed and stained after incubation following the manufacturer's specifications. The proliferation rate was calculated after normalization of the number of EdU-positive cells to the number of total nuclei (DAPI-stained cells) in five random fields.

For the cell cycle analysis, cells were trypsinized after $48 \mathrm{~h}$, washed twice with phosphate-buffered saline (PBS), and fixed in 75\% ethanol overnight. After washing twice with PBS, the cells were incubated with RNase A $(20 \mathrm{mg} / \mathrm{mL})$ at $37^{\circ} \mathrm{C}$ for 30 min, followed by staining with propidium iodide $(0.5 \mathrm{mg} / \mathrm{mL})$ at $4^{\circ} \mathrm{C}$ for $30 \mathrm{~min}$. Afterwards, cells were washed and resuspended in $500 \mu \mathrm{L}$ of PBS, and the DNA content was detected using a Becton Dickinson flow cytometer (BD Diagnostics, Sparks, MD).

\section{Reverse transcription quantitative PCR}

Total RNA was isolated from HUASMCs using RNA Bee Reagent (Cosmo Bio Inc., Tokyo, Japan). The total RNA was then prepared for first-strand cDNA synthesis by using the QuantiTect Reverse Transcription Kit (Qiagen Inc., Hilden, Germany). Reverse transcription quantitative PCR (RT-qPCR) was then performed using Thermal Cycler Dice TP800 (Takara Bio Inc., Kusatsu, Japan) with SYBR Premix Ex Taq II (Takara Bio Inc.), according to the manufacturer's instructions. Each cDNA sample was run in triplicate. The data were averaged, and standard deviations (SDs) were calculated. GAPDH was used as a standard control, and Table 1 describes the primers used in this study.

\section{Protein extraction}

Nuclear protein extraction was performed as follows. HUASMCs were lysed using buffer I (10 mM HEPES, $10 \mathrm{M}$ KCl, 1 M EDTA, 1 M M EGTA, 15\% Nonidet, protease inhibitors, and phosphatase

Table 1. List of oligonucleotides used for RT-qPCR

\begin{tabular}{lc}
\hline Primer & Sequence $\left(5^{\prime}-3^{\prime}\right)$ \\
\hline \multirow{2}{*}{ CYR61 } & Forward: CTGCGCTAACAACTCAACGA \\
& Reverse: GCAGATCCCTTTCAGAGCGG \\
CTGF & Forward: GGGCCTCTTCTGCGATTTC \\
& Reverse: ATCCAGGCAAGTGCATTGGTA \\
ANKRD1 & Forward: GGATGTGCCGAGGTTTCTGAA \\
& Reverse: GTCCGTTTATACTCATCGCAGAC \\
IL-1 $\beta$ & Forward: CTTCATGGTCCGTGGTACCGCCTGGAGTCT \\
& Reverse: TGCCCAGGGCATGGCACCAATCACCCTCTGAC \\
IL-6 & Forward: CTGATTGGAAACCTTATTAAG \\
& Reverse: CTGGTAGTATTACCTTCTTCA \\
IL-8 & Forward: GTAGCGAAGGACCTGTCAAACATTGGAAGATCA \\
& Reverse: CAACTCAGTGACCTTGCCTCCTCATGGTAATAC \\
GAPDH & Forward: AGGTCGGTGTGAACGGATTTG \\
& Reverse: TGTAGACCATGTAGTTGAGGTC \\
\hline
\end{tabular}




\section{Cellular Physiology Cell Physiol Biochem 2018;51:842-853

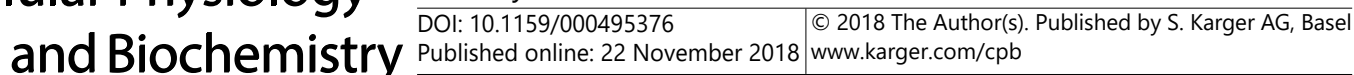 \\ Wang et al.: Arterial Wall Stress Induces Phenotypic Switching of Arterial Smooth \\ Muscle Cells}

inhibitors) and then centrifuged $\left(12000 \times \mathrm{g}, 4^{\circ} \mathrm{C}, 15 \mathrm{~min}\right)$. After centrifugation, the supernatant (cytosolic fraction) was collected. The remaining pellet, containing the nuclear fraction, was dissolved in $40 \mu \mathrm{L}$ of buffer II (20 mM HEPES, $400 \mathrm{mM} \mathrm{NaCl}, 10 \mathrm{mM}$ EDTA, $10 \mathrm{mM}$ EGTA, 15\% Nonidet, protease inhibitors, and phosphatase inhibitors). The resulting solution was then sonicated twice, each time for $5 \mathrm{~s}$, at $50 \mathrm{~W}$ and $4^{\circ} \mathrm{C}$. After centrifugation $\left(12000 \times \mathrm{g}, 4^{\circ} \mathrm{C}, 15 \mathrm{~min}\right)$, the supernatant containing the nuclear fraction was collected and stored at $-80^{\circ} \mathrm{C}$.

\section{Western blotting}

Protein samples were loaded onto a sodium dodecyl sulfate polyacrylamide gel (4-15\%) and run under standard conditions. The separated proteins were then transferred to polyvinylidene difluoride membranes (PVDF; Millipore, Billerica, MA). The membranes were blocked with 5\% nonfat milk at room temperature for $1 \mathrm{~h}$ in Tris-buffered saline with Tween-20 (TBST). Membranes were then incubated with primary antibodies against YAP (1:1000); p-YAP (1:1, 000; Ser 127); TAZ (1:1000); ICAM-1 (1:1000; CD54); VCAM-1 (1:1000); NFkB-p50 (1:1000); $\alpha$-smooth muscle actin (1:1000); smooth muscle myosin heavy chain (1:1000); calponin-1 (1:1000); Sav1 (1:1000); Lats1 (1:1000); PDK1 (1:1000); MST (1:1000) (all from Cell Signaling Technology); GAPDH (Abcam, Cambridge, UK), and histone (Abcam) overnight at $4^{\circ} \mathrm{C}$. After 3 washes with TBST, these membranes were then incubated for $60 \mathrm{~min}$ with peroxidase-conjugated Affinipure goat anti-rabbit IgG $(\mathrm{H}+\mathrm{L})$ and anti-mouse IgG $(\mathrm{H}+\mathrm{L})$-labeled secondary antibodies (both 1:2000). The membranes were washed in TBST with $0.5 \%$ Tween-20 before electrochemiluminescence (ECL) detection using BeyoECL Plus (Beyotime Institute of Biotechnology, Haimen, China). After ECL detection and exposure to X-ray film, the blots were stripped in $5 \mathrm{~mL}$ of stripping buffer (CoWin Biotech, Beijing, China) for 15 $\mathrm{min}$ at room temperature, followed by hybridization with an antibody against histones for normalization. Densitometric analysis of the resulting protein bands was conducted using the Tanon Gel Imaging System (Shanghai Tanon, Shanghai, China).

\section{Cell migration assay}

For the migration assay, the cells were resuspended at $1 \times 10^{6}$ cells $/ \mathrm{mL}$ after $24 \mathrm{~h}$ of starvation; 200 $\mu \mathrm{L}$ of cell suspension $\left(2 \times 10^{5}\right.$ cells) was seeded into the upper chambers (24-well insert), while medium supplemented with $10 \%$ FBS was placed in the lower chamber. After $48 \mathrm{~h}$ of incubation, the cells on the top side of the inserts were removed gently with a cotton swab. The inserts were then fixed with $4 \%$ methanol for $15 \mathrm{~min}$, followed by $0.1 \%$ crystal violet staining for $15 \mathrm{~min}$. The average migratory cells were counted by randomly choosing five fields for observation under a microscope, and this was repeated at least three times.

\section{Co-immunoprecipitation}

Cells were lysed with hypotonic buffer (10 mM Hepes at pH 7.4, $1 \mathrm{mM}$ EDTA, $150 \mathrm{mM} \mathrm{NaCl})$ supplemented with protease and phosphatase inhibitors. The resulting cell lysates were incubated with the PDK1 antibody for $2 \mathrm{~h}$ on ice, followed by precipitation with protein G Sepharose (Santa Cruz Biotechnology, Santa Cruz, CA). Samples were resolved by sodium dodecyl sulfate-polyacrylamide gel electrophoresis and transferred to PVDF membranes. After blocking with 5\% skim milk, the blots were incubated with antibodies for ECL detection.

\section{Statistical analysis}

Statistical analyses were performed using GraphPad Prism 5.0. Measurements are presented as the mean \pm SD. Comparisons between all pairs were performed using Student's $t$-tests or least significant difference tests, as appropriate. Comparisons among the means of multiple groups were performed by analysis of variance. The threshold for significance was $\mathrm{P}<0.05$. 


\section{Results}

Biomechanical stretch induced the activation and nuclear localization of YAP/TAZ in HUASMCs

To investigate whether YAP/TAZ are key molecules in VSMC phenotypic switching, the cultured HUASMCs were exposed to biomechanical stretch for $24 \mathrm{~h}$. YAP phosphorylation and YAP/ TAZ expression levels were then examined by western blotting, which demonstrated that biomechanical stretch led to a significant decrease in YAP phosphorylation and marked increases in YAP/TAZ expression. These findings suggested that biomechanical stretch resulted in the activation of YAP/TAZ expression in HUASMCs (Fig. 1A). Moreover, an RT-qPCR analysis revealed that the expression levels of YAP/ TAZ target genes, including CYR61, CTGF, and ANKRD1, increased significantly in response to biomechanical stretch (Fig. 1B). This finding demonstrated that stretching could be involved in inducing the activation and nuclear localization of YAP/TAZ, and thus the activation of genes involved in HUASMCmediated vascular remodeling.

\section{YAP/TAZ regulated HUASMC growth and cell cycle progression}

To investigate whether biomechanical stretch affected HUASMC growth, EDU labeling, and flow cytometry experiments were performed using cells treated with siRNAs against YAP/TAZ. Those cells were compared with cells that were not treated with siRNAs (siRNANC, or negative control cells). After transfection, western blotting revealed that YAP/TAZsiRNA-transfected cells had lower YAP/TAZ protein levels than those in siRNA-NC treated cells (Fig. 2A). Furthermore, the proliferation results based on EDU labeling suggested that proliferation was lower in YAP/TAZ-siRNA-transfected cells $(5.5 \pm 1.2 \%)$ than in siRNANC cells $(7.2 \pm 1.3 \%)$. In addition, YAP/TAZ-siRNA cells subjected to biomechanical stretch exhibited lower $(18.87 \pm 4.63 \%)$ proliferation levels than those of the biomechanical stretch group without YAP/TAZ siRNA treatment $(13.5 \pm 2.3 \%)$ (Fig. $2 \mathrm{~B})$.

Next, the DNA content was examined by flow cytometry, which showed that the biomechanical stretch group exhibited greater cell proliferation $(25.31 \pm 1.5 \%$, S phase $)$ than that in the non-stretched groups. By contrast, transfection of YAP/TAZ siRNA with or without biomechanical stretch decreased cell proliferation $(7.32 \pm 0.84 \%$ and $6.04 \pm 0.3 \%$, $\mathrm{S}$ phase, respectively) compared with that in the siRNA-NC-treated control group (11.14 $\pm 0.5 \%$, S phase) (Fig. $2 \mathrm{C}$ ). To evaluate the regulation of YAP in cell cycle progression, the expression levels of Cyclin A1 and proliferating cell nuclear antigen (PCNA) during the G1to-S transition were examined. Cyclin A1 and PCNA abundances were markedly increased in stretch-stimulated HUASMCs; these abundances decreased after treatment with YAP/ 


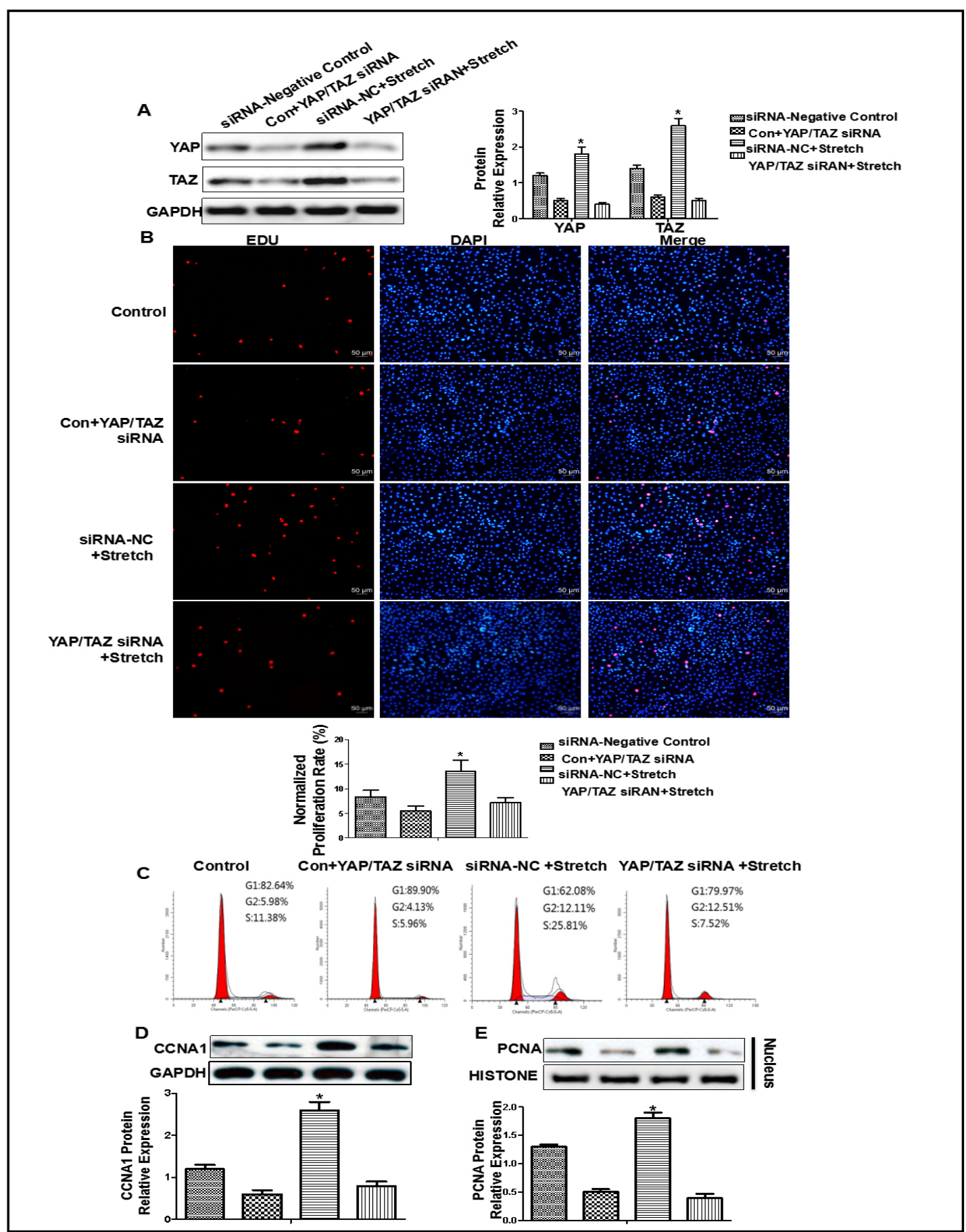

Fig. 2. YAP/TAZ regulated HUASMC growth and cell cycle progression (A) Western blot analysis of Yesassociated protein (YAP), and transcriptional co-activator with PDZ-binding motif (TAZ) protein expression in human arterial smooth muscle cells (HUASMCs) transfected with YAP/TAZ siRNA and subjected to biomechanical stretch. Protein expression was normalized to housekeeper protein GAPDH. (B) The cells were labeled with EdU to identify proliferative cells. Cell nuclei were stained with DAPI. (C) Percentage of of HUASMCs in different phases of the cell cycle after transfection with YAP/TAZ siRNA and subjected to biomechanical stretch. (D) Nuclear protein extraction and Western blot analysis of Cyclin A1 (CCNA1) and the proliferating cell nuclear antigen (PCNA) protein expression. Protein expression was normalized to GAPDH and HISTONE. Results shown were mean \pm SD from 5 independent experiments from each group tested. ${ }^{*} \mathrm{p}<0.05$ vs control.

\section{KARGER}




\section{Cellular Physiology Cell Physiol Biochem 2018;51:842-853 \begin{tabular}{ll|l|l|l} 
DOI: 10.1159/000495376 & O 2018 The Author(s). Published by S. Karger AG, Basel \\
and Biochemistry & Published online: 22 November 2018 www.karger.com/cpb \\
\cline { 2 - 4 }
\end{tabular} \\ Wang et al.: Arterial Wall Stress Induces Phenotypic Switching of Arterial Smooth \\ Muscle Cells}

TAZ siRNAs (Fig. 2D). These results suggested that biomechanical stretch was involved in programming HUASMCs toward a proliferative phenotype, and that YAP/TAZ played a role in proliferation, as decreased expression resulting from RNA interference led to reduced cell proliferation.

\section{YAP/TAZ activation promoted the stretch-induced pro-inflammatory response}

To investigate whether YAP/TAZ mediated the pro-inflammatory response in HUASMCs under biomechanical stretch, inflammatory cytokine gene expression was examined by RTqPCR. Tumor necrosis factor $\alpha$ expression was upregulated in stretch-induced HUASMCs and was decreased upon treatment with YAP/TAZ siRNAs (Fig. 3A). Cytokine mRNA levels between the two groups were also evaluated. Based on RT-qPCR, the levels of interleukin (IL)-1 $\beta$, IL-6, and IL-8 were significantly elevated in stretch-induced HUASMCs, and such increases were offset by YAP/TAZ siRNA treatment (Fig. 3B). The relationship between biomechanical stretch and NF- $\kappa B$ activation was also evaluated by examining NF- $\kappa B$ p50 expression level, which was up-regulated in the stretched group and decreased following YAP/TAZ knockdown (Fig. 3C). Additionally, since atherosclerosis is also involved monocyte attachment to HUASMCs, western blotting was used to examine the protein levels of the cell adhesion molecules VCAM-1 and ICAM-1. Biomechanical stretch up-regulated VCAM-1 and ICAM-1 levels in HUASMCs, while YAP/TAZ knockdown attenuated their expression, after likely induction by inflammatory cytokines (Fig. 3D). Taken together, these results indicated that wall stress may induce HUASMC inflammation, which in turn contributed to vascular remodeling and atherogenesis.

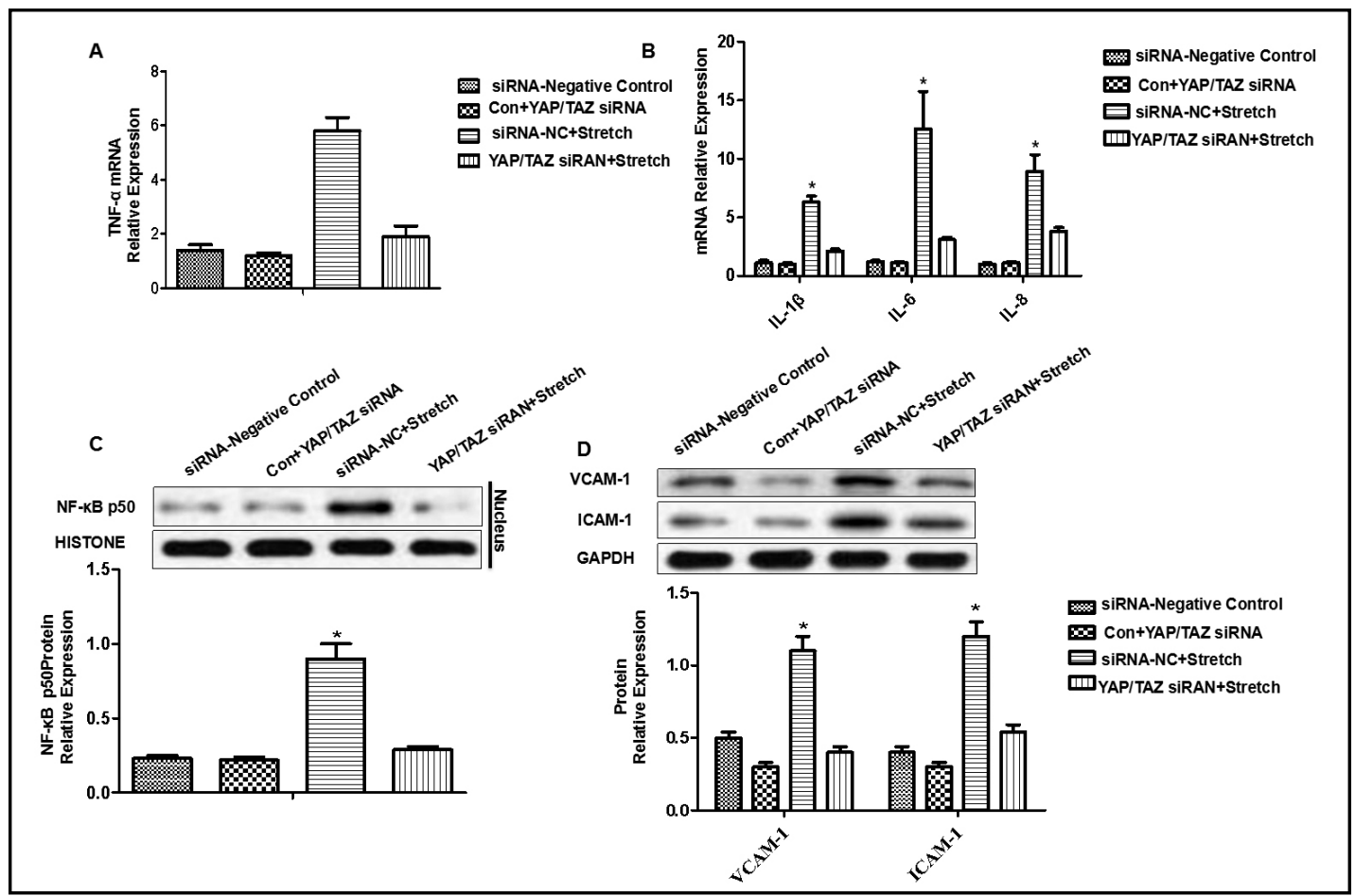

Fig. 3. YAP/TAZ activation promoted stretch-induced proinflammatory response (A) and (B) Real time -qPCR was used to determine the mRNA expression profiles of TNF- $\alpha$, IL-1 $1 \beta$, IL- 6 and IL-8 in human arterial smooth muscle cells (HUSMCs) subjected to biomechanical stretch and Yes-associated protein (YAP), and transcriptional co-activator with PDZ-binding motif (TAZ)-siRNA treatment. Expression was normalized to housekeeper mRNA GAPDH. Western blot analysis of nuclear protein NF-KB p50 (C) and cell adhesion molecules (D, VCAM-1, ICAM-1) from HUASMCs transfected with YAP/TAZ siRNA and subjected to biomechanical stretch. Protein expression was normalized to GAPDH and HISTONE. Results shown were mean \pm SD from 5 independent experiments from each group tested. ${ }^{*} \mathrm{p}<0.05$ vs control. 
Statin counteracted the stretch-induced phenotypic switch of HUASMCS

Statins,
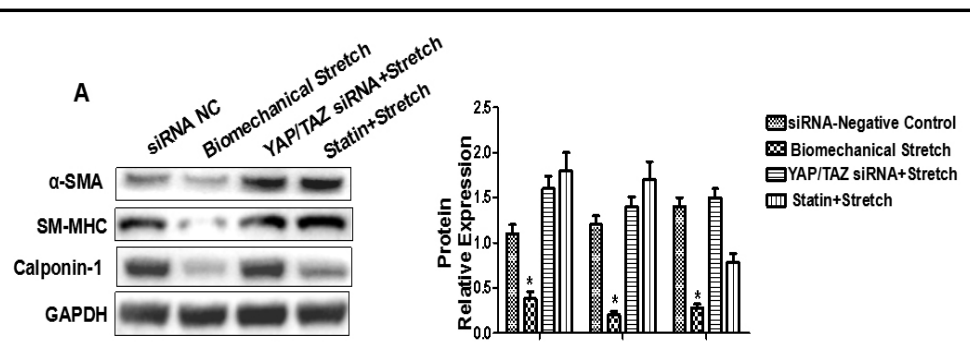

HMG-CoA reductase inhibitors, suppress YAP expression and prevent YAP-mediated transcription in tumors. Accordingly, we investigated whether YAP/TAZ mediated the statin-modulated phenotypic switch in HUASMCs. Protein expression levels of $\alpha$-SMA, SM-MHC, and calpoinin-1 in response to YAP/TAZ siRNAs or atorvastatin were examined. The $\alpha$-SMA, SM-MHC, and calponin-1 protein levels decreased B

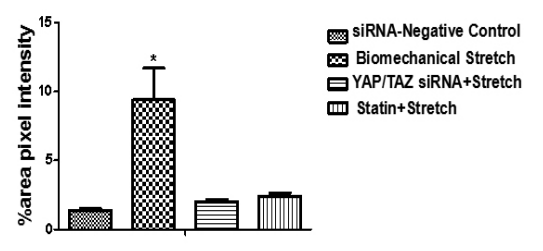

Fig. 4. Statin attenuated stretch-induced phenotypic switch of HUASMCs (A) Western blot analysis of whole cell lysates from human arterial smooth muscle cells (HUASMCs) subjected to statin and biomechanical stretch, with or without Yes-associated protein (YAP) and transcriptional co-activator with PDZ-binding motif (TAZ) siRNA treatment. Protein expression was normalized to housekeeper protein GAPDH. (B) Quantification of cell migration of HUASMCs exposed to statin and biomechanical stretch, with or without YAP/TAZ siRNA. Results shown were mean \pm SD from 5 independent experiments from each group tested. ${ }^{*} \mathrm{p}<0.05$ vs control.

significantly in response

to biomechanical stretch and were restored by YAP/TAZ siRNAs or atorvastatin (Fig. 4A).

With respect to the influence of biomechanical stretch on HUASMC migration, a Transwell plate was used to assay cell migration. Biomechanical stretch significantly increased HUASMC migration compared with that in the control group, and YAP/TAZ siRNA transfection or atorvastatin treatment abrogated stretch-induced HUASMC migration (Fig. 4B). Taken together, these results indicated that statin attenuated the stretch-induced phenotypic switch of HUSMCs from a contractile to a synthetic state by suppressing YAP/ TAZ expression and activity.

\section{Statins inhibited YAP/TAZ expression via PI3K and PDK1}

Several reports have demonstrated that angioplasty results in arterial wall stretch, leading to rapid mitogen-activated protein kinase (MAPK) activation in regenerating carotids. The role of biomechanical stretch in YAP/TAZ pathway mediation was further investigated using various specific pharmacologic inhibitors of its downstream pathways, including U0126 (mitogen-activated protein kinase inhibitor), SB203580, (p38 MAPK inhibitor), LY294002 (PI3K inhibitor) AKT inhibitor II (AKT inhibitor), and phosphoinositide-dependent kinase (PDK1) inhibitor II (PDK1 inhibitor). Western blotting showed that YAP and TAZ nuclear abundances were markedly decreased in stretch-stimulated HUASMCs upon the inhibition of PI3-kinase activity with LY294002. Moreover, an inhibitor of the PI3K downstream effector, PDK1, was also able to block YAP nuclear accumulation (Fig. 5). However, inhibiting AKT, another major kinase downstream of PI3K, had no effect on YAP nuclear accumulation. These results demonstrated that biomechanical stretch regulated YAP/TAZ activity in HUASMCs via the PI3K-PDK1 pathway.

\section{Interaction between PDK1 and the Hippo complex was mediated by Sav1}

The core components of the mammalian Hippo pathway include serine/threonine kinases Mst1/2 (Hippo/Hpo homologs), Lats1/2 (Warts/Wts homologs), and the adaptor proteins Sav1 (Salvador/Sav homolog) and Mob (Mats homologs). Some proteins of the 


\section{Cellular Physiology Cell Physiol Biochem 2018;51:842-853 \begin{tabular}{ll|l} 
and Biochemistry Published online: 22 November 2018 & $\begin{array}{l}\text { (c) } 2018 \text { The Author(s). Published by S. Karger AG, Basel } \\
\text { www.karger.com/cpb }\end{array}$ \\
\hline
\end{tabular} \\ Wang et al.: Arterial Wall Stress Induces Phenotypic Switching of Arterial Smooth}

Muscle Cells

Fig. 5. Statin inhibited YAP/TAZ expression through PI3K and PDK1, but independent of AKT Western blot analyses of whole cell lysates from human arterial smooth muscle cells (HUASMCs) treated with biomechanical stretch, statin and inhibitors (U0126, mitogen-activated protein kinase inhibitor; SB203580, p38 MAPK inhibitor; LY294002, PI3K inhibitor; AKT inhibitor II, AKT inhibitor; and phosphoinositide-dependent kinase [PDK1] Inhibitor II, PDK1 inhibitor). Cell lysates were immunoblotted by antibodies against YAP and TAZ. Protein expression was normalized to housekeeper protein GAPDH. Results shown were mean \pm SD from 5 independent experiments from each group tested. ${ }^{*} \mathrm{p}<0.05$ vs control; $\# \mathrm{p}<0.05$ vs biomechanical stretch.

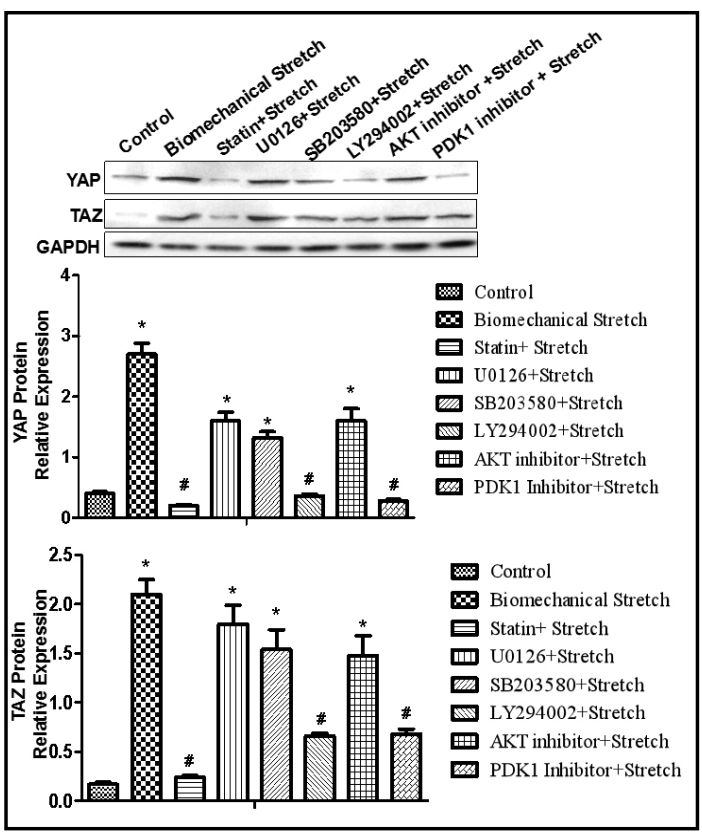

Hippo kinase cascade, including Mst, Sav1, and Lats, form a complex. To elucidate whether PDK1 was involved in the same complex as the Hippo pathway kinases, co-immunoprecipitation (co-IP) with endogenous PDK1 was used. Sav1, Mst, and Lats1 co-immunoprecipitated with endogenous PDK1. The interaction of PDK1 with these components was disrupted after $12 \mathrm{~h}$ of biomechanical stretch (Fig. 6A). However, pretreatment with a PI3K inhibitor and PDK1 inhibitor blocked the effects of stretch-induced complex dissociation. These results suggested that stretch-induced complex dissociation depended on PI3K-PDK1 pathway activity and PDK1 was a component of the Hippo pathway complex.

To further confirm the association of PDK1 in the Hippo complex, siRNA and coIP were used to examine the interaction between PDK1 and the Hippo complex. PDK1 activation triggered the dissociation of the Hippo core kinases from the scaffolding protein SAV1/WW45, leading to the inactivation of Lats, dephosphorylation, and YAP nuclear translocation. Thus, Sav1 may mediate the association between Lats and PDK1. Further analyses of the interaction between Mst and PDK1 showed that Mst silencing abrogated the Lats

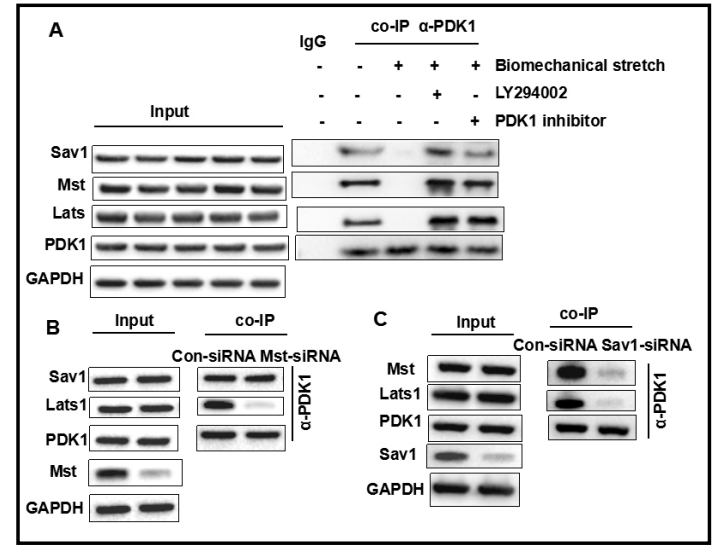

Fig. 6. Interaction between PDK1 and the Hippo complex was mediated through Sav1 (A) Cell lysates from human arterial smooth muscle cells (HUASMCs), subjected to biochemical stretch and inhibitors (LY294002, PI3K inhibitor; phosphoinositidedependent kinase (PDK1) Inhibitor II, PDK1 inhibitor), were co-immunoprecipitated with PDK1 antibody. The precipitated PDK1 immunocomplex input samples were subjected to Western blot analysis with the antibodies indicated. (B) and (C) Lysates from control siRNA or Mst/Sav1 siRNAtreated HUASMCs were incubated with anti-PDK1 antibody. As determined by co-immunoprecipitation, loss of Mst abrogated the binding between PDKLats. Moreover, loss of Sav1 abolished the PDK-Lats and PDK1-Mst interactions. Results shown were mean \pm SD from 3 independent experiments from each group tested. 


\section{Cellular Physiology Cell Physiol Biochem 2018;51:842-853 \begin{tabular}{ll|l|l|l|l} 
DOl: 10.1159/000495376 & 2018 The Author(s). Published by S. Karger AG, Basel \\
and Biochemistry & Published online:22 November 2018 www.karger.com/cpb
\end{tabular} \\ Wang et al.: Arterial Wall Stress Induces Phenotypic Switching of Arterial Smooth \\ Muscle Cells}

and PDK1 association. Moreover, the loss of Sav1 also abolished PDK-Lats and PDK1-Mst interactions (Fig. 6B and C). Thus, Sav1 was collectively shown to be the key molecule regulating the PDK1-Hippo complex interaction.

\section{Discussion}

Increases in wall stress and biomechanical stretch are a consequence of hypertension and are potent drivers of arterial remodeling. Our results indicated that YAP/TAZ expression in HUASMCs promotes cell proliferation and induces a phenotypic switch, and these effects are reversed upon silencing endogenous YAP/TAZ. Furthermore, we investigated whether YAP/TAZ is involved in mediating statin-modulated athero-protective effects. Our results proved that YAP/TAZ inactivation under statin treatment had inhibitory effects on cell proliferation and inflammation. Other studies have demonstrated that in stretch-stimulated VSMCs, various serine/threonine protein kinases, such as MAPK family proteins, are activated $[7,23,24]$. Indeed, our observation that YAP/TAZ activity is mediated by PI3K and PDK1 suggested that PI3K signaling activation inhibited the Hippo pathway. To further investigate the role of PDK1 in the Hippo complex, we examined the PDK1-Hippo interaction by co-IP. We discovered that the loss of Sav1 abolished PDK-Lats and PDK1-Mst interactions, suggesting that Sav1 is the key molecule regulating PDK1-Hippo interactions. YAP/TAZ, with a regulatory role in HUASMC inflammation and proliferation, may be involved in the induction of vascular remodeling.

In the vascular system, smooth muscle-specific YAP deletions lead to profound cardiac defects and vascular malformation [25]. The induction and activation of YAP expression following arterial injury deteriorates neointima formation and subsequent stenosis due to resulting VSMC proliferation [22]. We demonstrated that YAP/TAZ translocation results in significant changes in HUASMC growth, indicating its essential roles in proliferation and cell cycle progression. In addition, biomechanical stretch enhanced YAP/TAZ activation and induced a strong pro-inflammatory response in HUASMCs; YAP/TAZ silencing attenuated cell adhesion molecule and inflammatory cytokine expression. These findings indicated that YAP/TAZ activation may exert a proinflammatory effect on atherosclerotic lesions. LATS kinases canonically control YAP/TAZ activity via the regulation of YAP phosphorylation status and subsequent cytoplasmic/nuclear localization [26]. Moreover, mechanical stimuli adjust YAP/TAZ activity in a Rho-GTPases/actomyosin-dependent manner [27, 28]. Along with the Hippo and Rho pathways, other known mechano-sensors, such as G protein-coupled receptors, integrins $[17,29]$, AMP-activated protein kinase, and VE-cadherin-mediated PI3K/ AKT signaling [30, 31], regulate YAP/TAZ activity via phosphorylation. However, despite the crucial roles of PI3K and PDK1 in the growth factor regulation of Hippo signaling, AKT, a major effector kinase that acts downstream of these proteins, did not appear to be involved in Hippo pathway regulation in the present study. These findings suggested that alternative effectors may be present. It should be noted that even though PDK1 is known to act via other substrates, our results suggested that PDK1 directly controls the Hippo pathway [32]. However, the specific Hippo signaling pathway protein that is phosphorylated by PDK1 has not been clearly identified.

\section{Conclusion}

We demonstrated that YAP/TAZ serves as a critical mechano-transducer in the regulation of smooth muscle cell proliferation and inflammation. Therefore, the stretch-induced activation of YAP/TAZ may constitute a novel regulatory mechanism during stretch or wall stress-induced maladaptive remodeling processes in the early phases of atherosclerosis. 


\section{Cellular Physiology Cell Physiol Biochem 2018;51:842-853 \begin{tabular}{ll|l} 
and BiOChemistry & DOl: 10.1159/000495376 & $\begin{array}{l}\text { (c) } 2018 \text { The Author(s). Published by S. Karger AG, Basel } \\
\text { www.karger.com/cpb }\end{array}$ \\
\hline
\end{tabular} \\ Wang et al.: Arterial Wall Stress Induces Phenotypic Switching of Arterial Smooth Muscle Cells}

\section{Abbreviations}

Vascular (smooth muscle cells (VSMCs)); Yes-associated (protein (YAP)); transcriptional (co-activator with PDZ-binding motif (TAZ)); human (umbilical arterial smooth muscle cells (HUASMCs)); PI3-kinase ((PI3K); phosphoinositide dependent kinase (PDK1)); fetal (bovine serum (FBS)); proliferating (cell nuclear antigen (PCNA)); mitogen-activated (protein kinase (MAPK).

\section{Acknowledgements}

This study was supported by the National Natural Science Foundation of China (Grants No. 81800269 and 81800362) and the China Postdoctoral Science Foundation Grant (Grants No. 2014 M56137), Heilongjiang Postdoctoral Foundation Assistance (Grants No. LBH-Z14218), and Second Affiliated Hospital of Harbin Medical University Science Foundation (Grants No. KYBS2015-20).

\section{Disclosure Statement}

The authors declare that they have no competing interests.

\section{References}

$>1$ Owens GK, Kumar MS, Wamhoff BR: Molecular regulation of vascular smooth muscle cell differentiation in development and disease. Physiological reviews 2004;84:767-801.

-2 Humphrey JD: Mechanisms of arterial remodeling in hypertension: coupled roles of wall shear and intramural stress. Hypertension (Dallas, Tex : 1979) 2008;52:195-200.

-3 Hoefer IE, den Adel B, Daemen MJ: Biomechanical factors as triggers of vascular growth. Cardiovascular research 2013;99:276-283.

4 Baeyens N, Schwartz MA: Biomechanics of vascular mechanosensation and remodeling. Molecular biology of the cell 2016;27:7-11.

5 VanEpps JS, Vorp DA: Mechanopathobiology of atherogenesis: a review. Journal of Surgical Research 2007;142:202-217.

6 Li C, Xu Q: Mechanical stress-initiated signal transductions in vascular smooth muscle cells. Cellular signalling 2000;12:435-445.

7 Haga JH, Li Y-SJ, Chien S: Molecular basis of the effects of mechanical stretch on vascular smooth muscle cells. Journal of biomechanics 2007;40:947-960.

-8 Morrow D, Sweeney C, Birney YA, Cummins PM, Walls D, Redmond EM, Cahill PA: Cyclic strain inhibits Notch receptor signaling in vascular smooth muscle cells in vitro. Circulation research 2005;96:567-575.

-9 Qi YX, Qu MJ, Yan ZQ Zhao D, Jiang XH, Shen BR, Jiang ZL: Cyclic strain modulates migration and proliferation of vascular smooth muscle cells via Rho - GDI $\alpha$, Rac1, and p38 pathway. Journal of cellular biochemistry 2010;109:906-914.

10 Overholtzer M, Zhang J, Smolen GA, Muir B, Li W, Sgroi DC, Deng C-X, Brugge JS, Haber DA: Transforming properties of YAP, a candidate oncogene on the chromosome 11q22 amplicon. Proceedings of the National Academy of Sciences 2006;103:12405-12410.

11 Pan D: The hippo signaling pathway in development and cancer. Developmental cell 2010;19:491-505.

12 Steinhardt AA, Gayyed MF, Klein AP, Dong J, Maitra A, Pan D, Montgomery EA, Anders RA: Expression of Yesassociated protein in common solid tumors. Human pathology 2008;39:1582-1589.

-13 Dong J, Feldmann G, Huang J, Wu S, Zhang N, Comerford SA, Gayyed MF, Anders RA, Maitra A, Pan D: Elucidation of a universal size-control mechanism in Drosophila and mammals. Cell 2007;130:1120-1133. 


\section{Cellular Physiology Cell Physiol Biochem 2018;51:842-853 \begin{tabular}{ll|l} 
and Biochemistry & $\begin{array}{l}\text { DOI: 10.1159/000495376 } \\
\text { Published online: } 22 \text { November } 2018\end{array}$ & $\begin{array}{l}\text { C) } 2018 \text { The Author(s). Published by S. Karger AG, Basel } \\
\text { www.karger.com/cpb }\end{array}$ \\
\cline { 1 - 3 }
\end{tabular}}

Wang et al.: Arterial Wall Stress Induces Phenotypic Switching of Arterial Smooth

Muscle Cells

14 Yu F-X, Zhao B, Guan K-L: Hippo pathway in organ size control, tissue homeostasis, and cancer. Cell 2015;163:811-828.

15 Halder G, Dupont S, Piccolo S: Transduction of mechanical and cytoskeletal cues by YAP and TAZ. Nature reviews Molecular cell biology 2012;13:591.

16 Chaqour B, Goppelt-Struebe M: Mechanical regulation of the Cyr61/CCN1 and CTGF/CCN2 proteins. The FEBS journal 2006;273:3639-3649.

-17 Yu F-X, Zhao B, Panupinthu N, Jewell JL, Lian I, Wang LH, Zhao J, Yuan H, Tumaneng K, Li H: Regulation of the Hippo-YAP pathway by G-protein-coupled receptor signaling. Cell 2012;150:780-791.

-18 Taniguchi K, Wu L-W, Grivennikov SI, De Jong PR, Lian I, Yu F-X, Wang K, Ho SB, Boland BS, Chang JT: A gp130-Src-YAP module links inflammation to epithelial regeneration. Nature 2015;519:57.

-19 Fan R, Kim N-G, Gumbiner BM: Regulation of Hippo pathway by mitogenic growth factors via phosphoinositide 3-kinase and phosphoinositide-dependent kinase-1. Proceedings of the National Academy of Sciences 2013;110:2569-2574.

-20 Sorrentino G, Ruggeri N, Specchia V, Cordenonsi M, Mano M, Dupont S, Manfrin A, Ingallina E, Sommaggio R, Piazza S: Metabolic control of YAP and TAZ by the mevalonate pathway. Nature cell biology 2014;16:357.

21 Wang Z, Wu Y, Wang H, Zhang Y, Mei L, Fang X, Zhang X, Zhang F, Chen H, Liu Y: Interplay of mevalonate and Hippo pathways regulates RHAMM transcription via YAP to modulate breast cancer cell motility. Proceedings of the National Academy of Sciences 2014;111:E89-E98.

22 Wang X, Hu G, Gao X, Wang Y, Zhang W, Harmon EY, Zhi X, Xu Z, Lennartz MR, Barroso M: The induction of yes-associated protein expression after arterial injury is crucial for smooth muscle phenotypic modulation and neointima formation. Arteriosclerosis, thrombosis, and vascular biology 2012;32:2662-2669.

23 Zampetaki A, Zhang Z, Hu Y, Xu Q: Biomechanical stress induces IL-6 expression in smooth muscle cells via Ras/Rac1-p38 MAPK-NF-кB signaling pathways. American Journal of Physiology-Heart and Circulatory Physiology 2005;288:H2946-H2954.

24 Wang JG, Miyazu M, Matsushita E, Sokabe M, Naruse K: Uniaxial cyclic stretch induces focal adhesion kinase (FAK) tyrosine phosphorylation followed by mitogen-activated protein kinase (MAPK) activation. Biochemical and biophysical research communications 2001;288:356-361.

25 Wang Y, Hu G, Liu F, Wang X, Wu M, Schwarz JJ, Zhou J: Deletion of Yes-Associated Protein (YAP) Specifically in Cardiac and Vascular Smooth Muscle Cells Reveals a Crucial Role for YAP in Mouse Cardiovascular DevelopmentNovelty and Significance. Circulation research 2014;114:957-965.

26 Piccolo S, Dupont S, Cordenonsi M: The biology of YAP/TAZ: hippo signaling and beyond. Physiological reviews 2014;94:1287-1312.

27 Dupont S, Morsut L, Aragona M, Enzo E, Giulitti S, Cordenonsi M, Zanconato F, Le Digabel J, Forcato M, Bicciato S: Role of YAP/TAZ in mechanotransduction. Nature 2011;474:179.

28 Calvo F, Ege N, Grande-Garcia A, Hooper S, Jenkins RP, Chaudhry SI, Harrington K, Williamson P, Moeendarbary E, Charras G: Mechanotransduction and YAP-dependent matrix remodelling is required for the generation and maintenance of cancer-associated fibroblasts. Nature cell biology 2013;15:637.

29 Serrano I, McDonald PC, Lock F, Muller WJ, Dedhar S: Inactivation of the Hippo tumour suppressor pathway by integrin-linked kinase. Nature communications 2013;4:2976.

-30 Mo J-S, Meng Z, Kim YC, Park HW, Hansen CG, Kim S, Lim D-S, Guan K-L: Cellular energy stress induces AMPK-mediated regulation of YAP and the Hippo pathway. Nature cell biology 2015;17:500.

-31 Choi H-J, Zhang H, Park H, Choi K-S, Lee H-W, Agrawal V, Kim Y-M, Kwon Y-G: Yes-associated protein regulates endothelial cell contact-mediated expression of angiopoietin-2. Nature communications 2015;6:6943.

-32 Pearce LR, Komander D, Alessi DR: The nuts and bolts of AGC protein kinases. Nature reviews Molecular cell biology 2010;11:9. 